\title{
Vertical 2D Algebraic-Stress Turbulence Model of Shallow Water Flow in $\sigma$-Coordinates
}

\author{
S. M. Wong ${ }^{1 *}$, T. S. $\mathrm{Li}^{1}$, X. G. Wu ${ }^{1,2}$, Y. M. Shen ${ }^{3}$
}

${ }^{1}$ School of Science and Technology, the Open University of Hong Kong, Hong Kong, China

${ }^{2}$ Zhejiang Institute of Hydraulics \& Estuary, Hangzhou 310020 China

${ }^{3}$ State Key Laboratory of Coastal and Offshore Engineering, Dalian University of Technology, Dalian 116024 China

Email: anwong@ouhk.edu.hk

\begin{abstract}
:
A vertical 2D numerical model for shallow water flow is developed based on the unsteady Reynoldsaveraged Navier-Stokes equations. The equations are solved in the $\sigma$-coordinates system with semiimplicit time stepping. The eddy viscosity is calculated using algebraic-stress turbulence model. The boundary conditions at the bed for shallow water models only include the vertical diffusion terms using wall functions. The present model is used to simulate the water flow of $2 \mathrm{D}$ vertical plane through a steep-sided trench. The numerical results are in good agreement with the available experimental data.
\end{abstract}

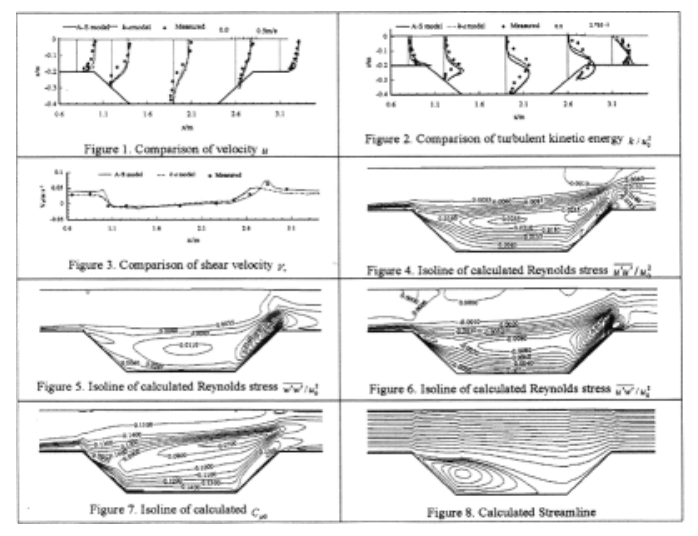

\section{REFERENCES}

1. Basara B, Younis B A. Prediction of turbulent flows in dredged trenches. J. Hydr. Res., 1996;34:813824

2. Li Z, Johns B. A numerical method for the determination of weakly non-hydrostatic non-linear free surface wave propagation. Int. J. Numer. Meth. Fluids, 2001;35:299-317

3. Stansby P K, Zhou J G. Shallow water flow solver with non-hydrostatic pressure: 2D vertical plane problem. Int. J. Numer. Meth. Fluids, 1998;28:541-563

4. Zhou J G, Stansby P K. An arbitrary Lagrangian-Eulerian $\sigma$ (ALES) model with non-hydrostatic pressure for shallow water flow. Comput. Meth. Appl. Mech. Eng., 1999;178:199-214

5. Wu X G. Turbulent model of flow and contamination convection-diffusion in curvilinear coordinates: [dissertation]. Dalian University of Technology, 2004

6. Rodi W. Turbulence Models and their Applications in Hydraulics. $3^{\text {rd }}$ Edition. IAHR, Delft, The Netherlands, 1993 